\title{
Contribution of normal aging to brain atrophy in MS
}

Christina J. Azevedo, MD, MPH, Steven Y. Cen, PhD, Amir Jaberzadeh, PhD, Ling Zheng, PhD, Stephen L. Hauser, MD, and Daniel Pelletier, MD

Neurol Neuroimmunol Neuroinflamm 2019;6:e616. doi:10.1212/NXI.0000000000000616

\section{Abstract}

\section{Objective}

To identify the top brain regions affected by MS-specific atrophy (i.e., atrophy in excess of normal aging) and to test whether normal aging and MS-specific atrophy increase or decrease in these regions with age.

\section{Methods}

Six hundred fifty subjects (2,790 MRI time points) were analyzed: 520 subjects with relapseonset MS from a 5-year prospective cohort with annual standardized 1-mm 3D T1-weighted images (3DT1s; 2,483 MRIs) and 130 healthy controls with longitudinal 3DT1s (307 MRIs). Rates of change in all FreeSurfer regions (v5.3) and Structural Image Evaluation Using Normalization of Atrophy (SIENA) were estimated with mixed-effects models. All FreeSurfer regions were ranked by the MS-specific atrophy slope/standard error ratio $\left(\beta_{\mathrm{MS}} \times\right.$ time $/ \mathrm{SE}_{\beta \mathrm{MS}} \times$ time $)$. In the top regions, age was added as an effect modifier to test whether MS-specific atrophy varied by age.

\section{Results}

The top-ranked regions were all gray matter structures. For SIENA, normal aging increased from $0.01 \% / y$ at age 30 years to $-0.31 \% / y$ at age 60 years $(-0.11 \% \pm 0.032 \% /$ decade, $p<0.01)$, whereas MS-specific atrophy decreased from $-0.38 \% / y$ at age 30 years to $-0.12 \% / y$ at age 60 years $(0.09 \% \pm 0.035 \% /$ decade, $p=0.01)$. Similarly, in the thalamus, normal aging increased from $-0.15 \% / y$ at age 30 years to $-0.62 \% / y$ at age 60 years $(-0.16 \% \pm 0.079 \% /$ decade, $p<$ $0.05)$, and MS-specific atrophy decreased from $-0.59 \% / y$ at age 30 years to $-0.05 \% / y$ at age 60 years $(0.18 \% \pm 0.08 \% /$ decade, $p<0.05)$. In the putamen and caudate, normal aging and MSspecific atrophy did not vary by age.

\section{Conclusions}

For SIENA and thalamic atrophy, the contribution of normal aging increases with age, but does not change in the putamen and caudate. This may have substantial implications to understand the biology of brain atrophy in MS.

\author{
Correspondence \\ Dr. Pelletier \\ Daniel.Pelletier@usc.edu
}

\section{RELATED ARTICLE}

\section{Editorial}

Untangling normal aging from disease-related brain atrophy in MS

Page e617

From the Department of Neurology (C.J.A., S.Y.C., A.J., L.Z., D.P.), University of Southern California, Los Angeles; and Department of Neurology (S.L.H.), University of California, San Francisco. 


\section{Glossary}

ADNI = Alzheimer's Disease Neuroimaging Initiative database $;$ CIS = clinically isolated syndrome; DMT = disease-modifying therapy; DTI = diffusion tensor imaging; HC = healthy control; PBVC = percentage whole-brain volume change; RRMS = relapsing-remitting MS; SIENA = Structural Image Evaluation Using Normalization of Atrophy; SPMS = secondary progressive MS; UCSF = University of California San Francisco.

The field of MS has devoted substantial effort to understanding and quantifying whole-brain and regional gray matter atrophy using MRI. Although the exact mechanism is unclear, brain atrophy in MS is thought to represent the net accumulation of tissue damage over time. Using MRI, numerous crosssectional $^{1,2}$ and longitudinal ${ }^{3,4}$ studies have demonstrated the clinical relevance of whole- and regional brain volume loss in MS. As such, brain volumetrics, particularly whole-brain volume, are often incorporated into MS clinical trials and clinical research as an in vivo proxy of neurodegeneration.

Historically, the literature describing brain atrophy in MS has generally reported the total volume loss in a given structure, e.g., whole-brain volume decline of $-0.7 \%$ to $-1.0 \%$ per year. ${ }^{1,4}$ Only recently has the contribution of normal aging begun to be explored, mainly in the context of developing "pathologic cutoff" values for whole-brain volume loss to discriminate patients with MS from healthy controls (HCs) ${ }^{5,6}$ with the ultimate goal of incorporating such values into a definition of "No Evidence of Disease Activity-4". However, a systematic, unbiased analysis that considers all brain regions to identify which regions undergo the highest amounts of excess atrophy in MS compared with normal aging has not been undertaken.

The excess atrophy in MS that is beyond normal aging, or "MS-specific atrophy," may be of fundamental importance because only the excess, disease-related atrophy is presumably modifiable with therapeutic intervention and should represent the therapeutic target for treatments aimed at slowing tissue loss in MS. This requires a robust understanding of normal aging, which is a complex process that varies across structures. $^{9-11}$ After normal head and brain growth during childhood, each brain structure declines with normal aging. The age at which the decline begins varies widely across structures, and the trajectory of decline may be linear or nonlinear depending on the structure. ${ }^{9-11}$ For example, whole-brain atrophy follows a nonlinear trajectory that accelerates significantly with age. ${ }^{6,12}$ Because the rate and trajectory of normal aging decline varies across structures, the amount of MS-specific atrophy should also vary across brain structures and as patients age.

The objectives of this analysis are to (1) identify the top brain regions that undergo the largest, least variable amount of MSspecific atrophy, and (2) test whether the amount of normal aging (and therefore the amount of MS-specific atrophy) increases or decreases in the top regions with age.

\section{Methods}

\section{Study population}

As described previously, ${ }^{8}$ from January 2005 through December 2010, all Caucasian patients aged 18-65 years who fulfilled the $2005 \mathrm{McD}$ onald Criteria ${ }^{13}$ for clinically isolated syndrome (CIS) or MS were offered enrollment into a large, prospective phenotype-genotype biomarker study (the EPIC study) at the University of California San Francisco (UCSF) MS Center. More than 500 subjects with CIS/MS were followed longitudinally with annual standardized clinical visits and $3 \mathrm{~T}$ brain MRIs for up to 5 years. All EPIC participants with relapse-onset $\mathrm{MS}^{14}$ were included in this analysis.

To increase the HC sample size, 2 HC data sets were pooled for this analysis. The first was a convenience sample of $89 \mathrm{HCs}$ (155 MRI time points) from UCSF, recruited separately from the EPIC cohort, but with brain MRIs performed using the same 3T MRI scanner and protocol as EPIC. In addition, the publicly available Alzheimer's Disease Neuroimaging Initiative database (ADNI; ida.loni.usc.edu) was queried to select ADNI HCs who had (1) a 3T 3D T1-weighted volumetric sequence, (2) longitudinal data ( $\geq 3$ time points), and (3) age 55-70 years. This yielded an additional $41 \mathrm{HCs}$ (152 MRI time points), for a total of $130 \mathrm{HCs}$ and $307 \mathrm{MRI}$ time points.

\section{Standard protocol approvals, registrations, and patient consents}

Both the EPIC study protocol and the UCSF HC study protocol were approved by the Committee on Human Research at UCSF, and informed consent was obtained from all participants. MRIs from ADNI (ClinicalTrials.gov identifier: NCT00106899) are publicly available.

\section{MRI acquisition}

All brain MRI scans from UCSF were acquired using an 8-channel phased array coil in reception and a body coil in transmission on a 3T GE Excite scanner that did not undergo hardware upgrades during the 5-year study period. A 3D, T1weighted, $1 \mathrm{~mm}$-isotropic, volumetric, inversion recovery spoiled gradient echo (3DT1) sequence $\left(1 \times 1 \times 1 \mathrm{~mm}^{3}, 180\right.$ slices) was acquired at each time point (Echo Time/ Repetition Time/Inversion Time $=2 / 7 / 400$ milliseconds, flip angle $=15^{\circ}, 256 \times 256 \times 180$ matrix, $240 \times 240 \times 180 \mathrm{~mm}^{3}$ field of view, number of excitations $=1$ ).

ADNI (adni-info.org) is a multicenter, multinational, NIHfunded cohort study focused on identifying and validating biomarkers in Alzheimer disease. All ADNI subjects are 
scanned using a standardized brain MRI protocol at $3 \mathrm{~T}$ after harmonization and optimization of pulse sequences across centers using a phantom. Per ADNI requirements, MRI scanners must be GE, Siemens, or Phillips systems with a 32channel coil, and subjects scanned longitudinally at a given site are required to go back to the same scanner, which is an accepted research practice to reduce measurement variability in longitudinal MRI studies. ${ }^{15}$ At each time point, a 3DT1 is acquired in sagittal plane with $1 \times 1 \times 1.2 \mathrm{~mm}$ voxel size. Each scan is reviewed to ensure that the specifications of the ADNI protocol are met, and a single phantom scan is obtained each day that an ADNI participant is scanned. Detailed ADNI procedures and protocols are available online (ida.loni. usc.edu).

\section{MRI postprocessing}

All 3DT1s were processed using our custom in-house pipeline, which has been described elsewhere ${ }^{8}$ and uses FreeSurfer's longitudinal processing stream ${ }^{16}$ (v5.3; surfer.nmr. mgh.harvard.edu/fswiki/LongitudinalProcessing), lesion inpainting, ${ }^{17}$ and manual edits as needed. We extracted final output from all available 119 brain regions labeled by FreeSurfer, including subcortical, CSF, ventricular, white matter, and whole-brain volumes, and cortical thickness measurements. In addition, annual percentage whole-brain volume change (PBVC) was calculated from 3DT1s using Structural Image Evaluation Using Normalization of Atrophy ${ }^{18}$ (SIENA; fsl.fmrib.ox.ac.uk/fsl/fslwiki/SIENA).

\section{Statistical analysis}

\section{Estimating change over time in each brain region}

All statistical analyses were performed in SAS (v9.4). $\alpha=$ 0.05 was used for all hypothesis testing. Paired left and right volumes were summed, and left and right gyral thickness measurements were averaged, yielding 83 total FreeSurfer regions for analysis. Regional volumes were standardized to percent of intracranial volume. For each brain region, including PBVC from SIENA, percent change from the initial scan was calculated at each time point. The change per year (atrophy slope) in each brain region was estimated in a linear mixed-effects model ${ }^{19}$ as follows: $Y_{i j}=\beta_{00}+\beta_{10}(\text { Time })_{i j}+$ $\beta_{01} M S+\beta_{11}(M S) *(\text { Time })_{i j}+b_{0 j}+b_{1 j}(\text { Time })_{i j}+\varepsilon_{i j}{ }^{20}$. This model includes fixed effects for on-study time (in years; $\beta_{10}(\text { Time })_{i j}$ ), case-control status (binary; $\beta_{01} M S$ ), the interaction between disease status and time $\left(\beta_{11}(M S) *(\text { Time })_{i j}\right)$, which estimates the additional brain atrophy in MS beyond normal aging (i.e., MS-specific atrophy), and random effects for intercept and slope $\left(b_{0 j}\right.$ and $b_{1 j}(\text { Time })_{i j}$, respectively). Assumptions of the statistical models were checked via residual plots to examine the model integrity, normality of residuals, and constant variance, as well as Cook influence plot to detect potential outliers with a strong influence on model estimates.

\section{Ranking brain regions}

From the linear mixed-effects model in each region, we extracted the parameter estimate and standard error for the interaction term $\beta_{11}(M S) *(\text { Time })_{i j}$, created a ratio $\frac{\beta_{11}(M S) *\left(\text { Time }_{i j}\right.}{S E\left[\beta_{11}(M S) *\left(\text { Time }_{i j}\right]\right.}$, and ranked all 83 FreeSurfer regions according to this ratio. This ratio identifies regions that undergo the highest amount of MS-specific atrophy over time with the least variability in the estimated change (i.e., the lowest standard error). Brain regions were ranked in unadjusted models, after adjusting for age, and after adjusting for both age and sex. Age and sex adjustments were performed by adding age $+\operatorname{age} *(\text { Time })_{i j}$ and sex $+\operatorname{sex} *(\text { Time })_{i j}$ to the model as fixed effects.

\section{Effect of age on normal aging and MS-specific atrophy}

The effect of age at study entry on normal aging and MSspecific atrophy was explored in the top-ranked FreeSurfer regions and PBVC from SIENA. To test whether normal aging and MS-specific atrophy change with age, age was modeled as an effect modifier in the relationship between MSspecific atrophy and time by adding a 3-way interaction term plus additional marginal termsage + age $* M S+$ age $*(\text { Time })_{i j}+$ age*MS*(Time $\left.)_{i j}\right)$ to the model listed above. Using the full model, atrophy slopes for subjects with MS (total atrophy in MS), HCs (normal aging), and the slope difference between MS and HCs (MS-specific atrophy) were estimated at ages 30, 40,50 , and 60 years. These ages were chosen a priori to represent the age range of the subjects with MS in our data set.

\section{MRI scanner as a potential confounder}

Because our data set was assembled from multiple sources, we tested for potential confounding by testing the interaction between MRI scanner manufacturer/model and atrophy slope $\left(\right.$ i.e. $\quad$ scanner $\left.*(\text { Time })_{i j}\right)$. Visual inspection was used to compare the ranking of brain regions and the point estimate of atrophy slopes at different age categories before and after adding scanner $*(\text { Time })_{i j}$ to the model.

\section{Data availability}

Two of the 3 data sets used in this analysis (the EPIC MS data set and the UCSF HC data set) are not sharable publicly. The acquisition of the brain MRI images in the EPIC MS data set was funded by investigator-initiated studies sponsored by pharmaceutical companies. The third data set is publicly available via the $\mathrm{ADNI}$ at ida.loni.usc.edu.

\section{Results}

\section{Subject characteristics}

Table 1 provides characteristics of the subjects included in this analysis. Six hundred fifty subjects and 2,790 MRI time points were analyzed, including 520 subjects with MS (2,483 MRI time points) and $130 \mathrm{HCs}$ (307 MRI time points). Of the 90 subjects who were classified as CIS at study entry, 47 (52.2\%) were reclassified as relapsing-remitting MS (RRMS) or secondary progressive MS (SPMS) by their treating physician during the study period. Of the 392 subjects who were classified as RRMS at study entry, 21 (5.4\%) were reclassified as SPMS by their treating physician while on study. Four hundred four subjects ( $77.7 \%$ of the cohort) were exposed to 
Table 1 Subject demographics and characteristics

\begin{tabular}{|c|c|c|}
\hline Variable & Subjects with MS $(n=520)^{a}$ & $\operatorname{HCs}(n=130)^{a}$ \\
\hline Age at study entry, y & $42.7 \pm 9.8$ & $47.9 \pm 15.1$ \\
\hline \multicolumn{3}{|l|}{ Age quartiles } \\
\hline $18-35$ & $129(24.7)$ & $34(26.4)$ \\
\hline $36-43$ & $143(27.6)$ & $21(16.3)$ \\
\hline $44-52$ & $142(27.4)$ & $22(17.1)$ \\
\hline $53-69$ & $106(20.4)$ & $52(40.3)$ \\
\hline \multicolumn{3}{|l|}{ Sex } \\
\hline Female & $365(70.2)$ & $83(63.8)$ \\
\hline Male & $155(29.8)$ & $47(36.2)$ \\
\hline Disease course at study entry & & N/A \\
\hline CIS & $90(17.3)$ & \\
\hline RRMS & $392(75.4)$ & \\
\hline SPMS & $38(7.3)$ & \\
\hline Clinical disease duration at study entry, y & $9.2 \pm 8.6$ & N/A \\
\hline Mean EDSS score at study entry & $1.8 \pm 1.5$ & N/A \\
\hline \multicolumn{3}{|l|}{ Cumulative DMT exposure $^{b}$} \\
\hline None & $116(22.3)$ & \\
\hline$\leq 1 \mathrm{y}$ & $33(6.3)$ & \\
\hline $1-3 y$ & $77(14.8)$ & \\
\hline $3-5 y$ & $107(20.6)$ & \\
\hline$>5 y$ & $187(36.0)$ & \\
\hline Mean follow-up time, $y$ & $4.1 \pm 1.6$ & $1.4 \pm 1.5$ \\
\hline Total MRI time points analyzed & 2,483 & 307 \\
\hline \multicolumn{3}{|l|}{ No. of MRI time points } \\
\hline Subjects with 1 MRI & 18 & 49 \\
\hline Subjects with 2 MRIs & 47 & 25 \\
\hline Subjects with 3 MRIs & 50 & 24 \\
\hline Subjects with 4 MRIs & 49 & 24 \\
\hline Subjects with 5 MRIs & 111 & 8 \\
\hline Subjects with 6 MRIs & 245 & - \\
\hline
\end{tabular}

Abbreviations: $\mathrm{CIS}=$ clinically isolated syndrome; DMT = disease-modifying therapy; EDSS = Expanded Disability Status Scale; HC = healthy control; RRMS = relapsing-remitting MS; SPMS = secondary progressive MS.

a Mean \pm SD for continuous variables, $\mathrm{N}(\%)$ for categorical variables.

${ }^{\mathrm{b}}$ Cumulative DMT exposure includes all DMT exposure that occurred prestudy and/or on study for each subject (i.e., throughout their lifetime).

disease-modifying therapy (DMT), the vast majority of which were injectable DMTs given the study period (2005-2010).

\section{Ranking of brain regions}

The top 5 FreeSurfer regions ranked by the slope/standard error ratio are given in table 2 . Putamen volume, subcortical gray matter volume, caudate volume, cuneus gyrus thickness, and thalamic volume were consistently ranked as the top 5 regions across all mixed-effects models, including the unadjusted model, after adjusting for age, and after adjusting for age and sex. Examination of residual plots showed a normal distribution of residuals and did not indicate any deviations 
Table 2 Top 5 brain regions ranked by slope/standard error ratio*

\begin{tabular}{llll}
\hline FreeSurfer region & Unadjusted & Age adjusted & Age and sex adjusted \\
\hline Putamen volume & -5.05 & -4.74 & -4.68 \\
\hline Subcortical gray matter volume & -4.11 & -3.44 & -3.44 \\
\hline Caudate volume & -4.13 & -3.33 & -3.4 \\
\hline Cuneus gyrus thickness & -3.0 & -2.64 & -2.64 \\
\hline Thalamic volume & -1.67 & -1.67 & -1.75 \\
\hline
\end{tabular}

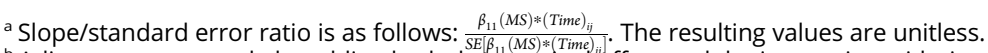

${ }^{\mathrm{b}}$ Adjustment was made by adding both the marginal effect and the interaction with time, e.g., age + age $\times$ time.

from a linear form, with relatively constant variance across the fitted range. The Cook influence plot did not show any observations with Cook distance $>1$ to indicate an influential outlier.

\section{Effect of age on MS-specific atrophy}

The contributions of MS and normal aging to total atrophy in the putamen, caudate, thalamus, and whole-brain volume (PBVC from SIENA) at each decade from age 30 to 60 years are displayed in the figure, with corresponding values in table 3. "Subcortical gray matter volume" in FreeSurfer includes the thalamus, caudate, hippocampus, amygdala, nucleus accumbens, ventral diencephalon, and substantia nigra (surfer.nmr.mgh. harvard.edu/fswiki/MorphometryStats). Because our aim was to analyze the effect of age on MS-specific atrophy in separate brain regions, subcortical gray matter volume was not retained for further analysis. Cuneus gyral thickness was also not retained because, unlike the deep gray matter nuclei, which are known to be affected by $M S^{21,22}$ there is a paucity of previous data suggesting specific involvement of the cuneus gyrus.

As shown in figure, A and table 3, the total whole-brain atrophy slope (change/y) in subjects with MS from SIENA was

Table 3 MS-specific and normal aging atrophy rates by region and decade

\begin{tabular}{|c|c|c|c|c|c|}
\hline Brain region & Age $30 y$ & Age $40 y$ & Age $50 y$ & Age $60 \mathrm{y}$ & $p$ Value \\
\hline \multicolumn{6}{|l|}{ SIENA } \\
\hline Total atrophy $(\% / y)$ & $-0.37 \pm 0.02$ & $-0.39 \pm 0.01$ & $-0.41 \pm 0.02$ & $-0.43 \pm 0.03$ & \\
\hline MS-specific atrophy & $-0.38 \pm 0.09$ & $-0.29 \pm 0.06$ & $-0.21 \pm 0.05$ & $-0.12 \pm 0.06$ & 0.01 \\
\hline Normal aging & $0.01 \pm 0.09$ & $-0.10 \pm 0.06$ & $-0.21 \pm 0.05$ & $-0.31 \pm 0.05$ & $<0.01$ \\
\hline \multicolumn{6}{|l|}{ Thalamus } \\
\hline Total atrophy $(\% / y)$ & $-0.74 \pm 0.05$ & $-0.71 \pm 0.03$ & $-0.69 \pm 0.04$ & $-0.67 \pm 0.07$ & \\
\hline MS-specific atrophy & $-0.59 \pm 0.22$ & $-0.41 \pm 0.15$ & $-0.23 \pm 0.12$ & $-0.05 \pm 0.15$ & $<0.05$ \\
\hline Normal aging & $-0.15 \pm 0.21$ & $-0.31 \pm 0.15$ & $-0.46 \pm 0.12$ & $-0.62 \pm 0.13$ & $<0.05$ \\
\hline \multicolumn{6}{|l|}{ Caudate } \\
\hline Total atrophy $(\% / y)$ & $-1.31 \pm 0.07$ & $-1.19 \pm 0.04$ & $-1.07 \pm 0.05$ & $-0.96 \pm 0.08$ & \\
\hline MS-specific atrophy & $-0.67 \pm 0.26$ & $-0.59 \pm 0.19$ & $-0.52 \pm 0.15$ & $-0.44 \pm 0.18$ & 0.48 \\
\hline Normal aging & $-0.64 \pm 0.26$ & $-0.60 \pm 0.18$ & $-0.56 \pm 0.14$ & $-0.51 \pm 0.16$ & 0.67 \\
\hline \multicolumn{6}{|l|}{ Putamen } \\
\hline Total atrophy $(\% / y)$ & $-1.53 \pm 0.08$ & $-1.48 \pm 0.05$ & $-1.42 \pm 0.06$ & $-1.37 \pm 0.10$ & \\
\hline MS-specific atrophy & $-1.05 \pm 0.34$ & $-0.99 \pm 0.24$ & $-0.93 \pm 0.19$ & $-0.87 \pm 0.24$ & 0.66 \\
\hline Normal aging & $-0.49 \pm 0.33$ & $-0.49 \pm 0.23$ & $-0.50 \pm 0.18$ & $-0.50 \pm 0.22$ & 0.97 \\
\hline
\end{tabular}

Abbreviations: $\mathrm{HC}=$ healthy control; SIENA = Structural Image Evaluation using Normalization of Atrophy.

Point estimates \pm standard error for atrophy rates (percent change per year) in the top brain regions at ages 30,40,50, and $60 \mathrm{y}$ for the total rate of atrophy observed in patients with MS ("total atrophy"), the rate of atrophy observed in HCs (i.e., normal aging), and the rate of MS-specific atrophy (slope for MS-slope for $\mathrm{HC}$ ), estimated with linear mixed-effects models in each region. The $p$ value given refers to the test of difference in normal aging and MS-specific atrophy rates between consecutive decades, with the null hypothesis that the difference between consecutive decades equals zero. 

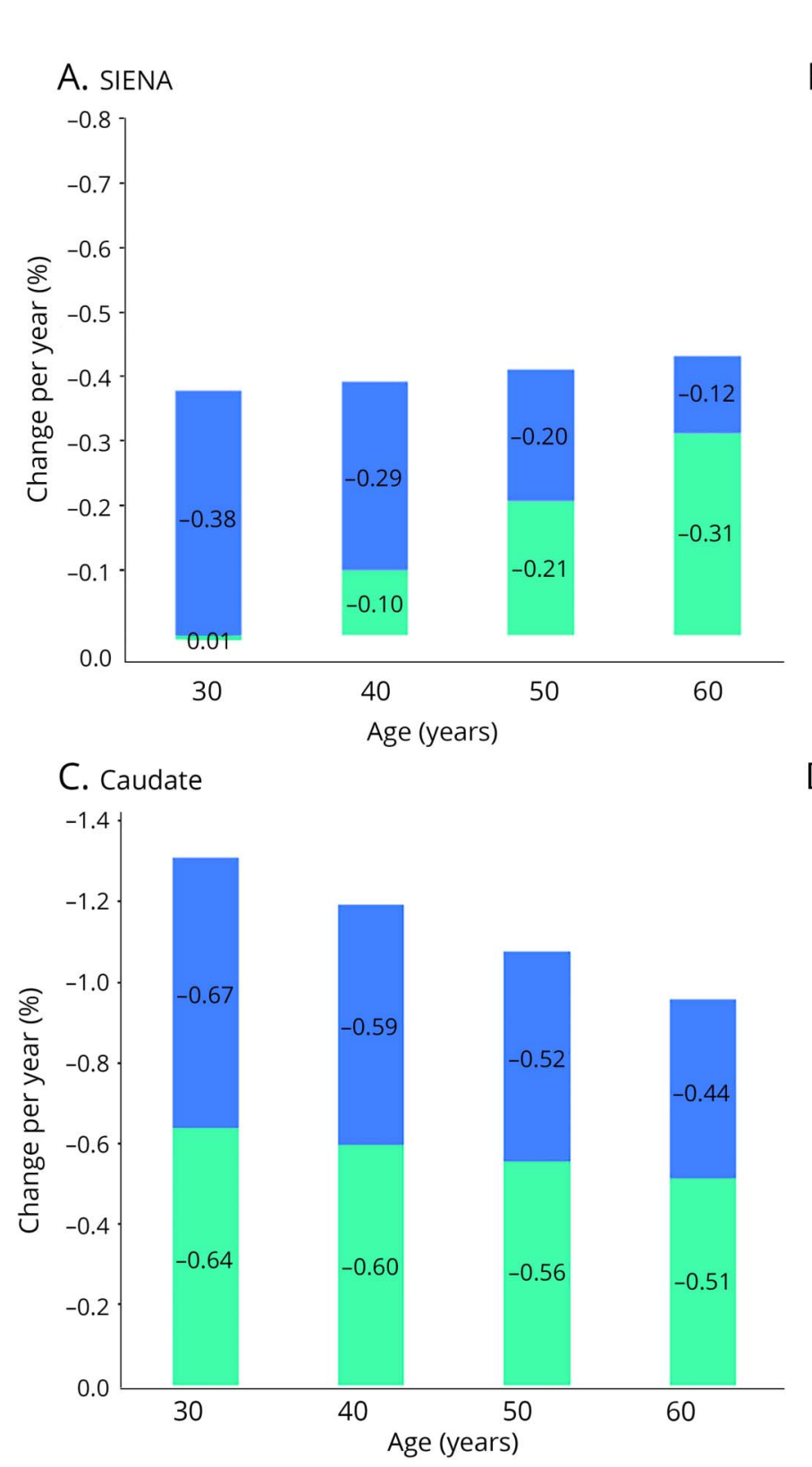

Normal aging

- MS-specific atrophy
B. Thalamus
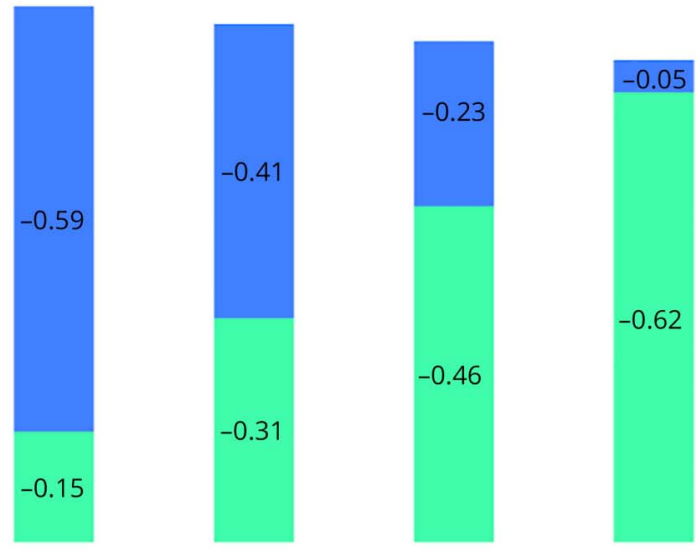

30

40

50

60

D. Putamen

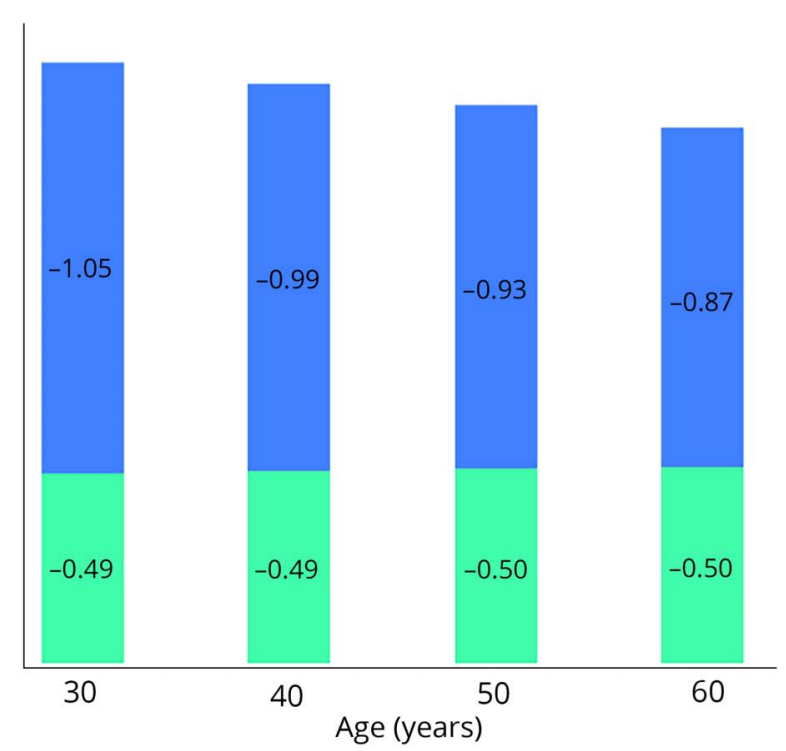

Stacked histograms showing the trend of brain atrophy slopes by age in HCs (green) and MS-specific atrophy (blue). The total rate of atrophy in patients with MS is represented by the total height of each histogram bar (combining colors). For SIENA (A) and thalamus (B), the contribution of MS-specific atrophy and normal aging to the total atrophy slope changed significantly across decades, whereas normal aging was stable across decades in the caudate (C) and putamen (D). HC = healthy control; SIENA = structural image evaluation using normalization of atrophy.

approximately $-0.4 \%$ per year, consistent across decades. However, the whole-brain atrophy slopes for MS-specific atrophy and normal aging changed significantly with age. The rate of MS-specific whole-brain atrophy decreased from $-0.38 \%$ per year at age 30 years to $-0.12 \%$ per year at age 60 years, a change of $0.09 \% \pm 0.035 \%$ per decade $(p=0.01)$, whereas the normal aging atrophy slope increased from $0.01 \%$ per year at age 30 years to $-0.31 \%$ per year at age 60 years, a change of $-0.11 \% \pm 0.032 \%$ per decade $(p<0.01)$. A similar pattern was observed in the thalamus (figure, B and table 3 ). In the thalamus, the total rate of atrophy in subjects with MS was approximately $-0.7 \%$ per year, consistent across decades. However, the rate of normal aging atrophy accelerated from $-0.15 \%$ per year at age 30 years to $-0.62 \%$ per year at age 60 years $(-0.16 \% \pm 0.079 \%$ per decade, $p<0.05)$, whereas the rate of MS-specific atrophy decreased from $-0.59 \%$ per year at age 30 years to $-0.05 \%$ per year at age 60 years $(0.18 \% \pm$ $0.08 \%$ per decade, $p<0.05$ ).

A very different pattern was observed in the putamen and caudate (figure, $\mathrm{C}$ and $\mathrm{D}$ and table 3 ). In these regions, the contributions of normal aging and MS-specific atrophy did 
not vary significantly with age. In the caudate, the normal aging and MS-specific atrophy slopes were stable across decades, with nonsignificant changes of $0.04 \pm 0.10(p=0.67)$ and $0.07 \pm 0.11(p=0.48)$ per decade, respectively. Similarly, in the putamen, the rate of normal aging $(-0.01 \% \pm 0.13 \%, p=$ $0.97)$ and MS-specific atrophy $(0.06 \% \pm 0.14 \%, p=0.66)$ did not change significantly across decades.

\section{Type of MRI scanner as a potential confounder} None of the interaction tests between the type of MRI scanner and the atrophy slope $\left(\right.$ scanner $*\left(\right.$ Time $\left._{i j}\right)$ were statistically significant. The rankings and parameter estimates did not change after adding scanner* $(\text { Time })_{i j}$ to the model, suggesting that scanner manufacturer/model did not confound our findings.

\section{Discussion}

We used an agnostic approach to identify the top brain regions with the highest, least variable amount of yearly MSspecific atrophy in a large, 5-year, prospective cohort of subjects with MS and estimated the contribution of normal aging to atrophy measurements in the top-ranked regions at each decade from ages 30 to 60 years. Out of every region in the human brain that is labeled by FreeSurfer, all the topranked regions were gray matter structures, including 3 deep gray nuclei and 1 cortical gyrus. This is consistent with previous histopathologic and MRI studies suggesting involvement of deep and cortical gray matter in the MS disease process $^{21-23}$ and underscores the importance of gray matter in MS.

More importantly, our findings indicate that for whole-brain (SIENA) and thalamic volume, the contribution of normal aging to total atrophy may increase significantly as the MS population ages. In these regions, most of the atrophy we observed at age 30 years was clearly in excess of normal aging and presumably disease related, whereas by age 60 years, the rate of normal aging had accelerated such that there was little excess atrophy attributable to MS. In the caudate and putamen, on the other hand, we observed a stable contribution of normal aging at each decade. In the case of whole-brain atrophy, our observed acceleration of normal aging is consistent with existing literature ${ }^{12}$ and has been observed in other studies using SIENA. ${ }^{5,6}$ Although normal aging in subcortical structures is perhaps less well characterized, a similar pattern of behavior could plausibly occur. Some subcortical structures such as the amygdala, caudate, and thalamus have been shown to follow a linear decline over the lifespan in normal aging, whereas others such as the hippocampus may follow a quadratic (i.e., nonlinear) trajectory. ${ }^{11}$ Most of the studies modeling atrophy in subcortical structures in normal aging have used cross-sectional data sets composed of 1.5T MRIs and relatively simplified statistical methods (i.e., linear or quadratic age terms) to estimate the effect of age. ${ }^{9-11}$ Our data sets were composed of longitudinal $3 \mathrm{~T}$ MRIs, and we used a more complex modeling strategy, making direct comparisons with published literature somewhat difficult.

We found that different brain regions behaved differently with respect to MS-specific atrophy vs normal aging. This suggests that tissue loss in MS may occur for reasons that are distinct across brain regions. Axonal transection within white matter demyelinating lesions ${ }^{24}$ can lead to upstream or downstream neurodegeneration, which likely contributes to whole-brain and thalamic atrophy in MS. ${ }^{25,26}$ This may be a particularly important mechanism of thalamic atrophy, which is an area of early neurodegeneration in MS. ${ }^{26-29}$ A strong preponderance for MS lesions to form in thalamocortical white matter and a probable mechanistic link between thalamocortical white matter lesions and thalamic atrophy has been demonstrated using diffusion tensor imaging (DTI) tractography in subjects with CIS. ${ }^{26}$ We hypothesize that the downstream effects of white matter lesions may explain the predominantly diseaserelated atrophy we observed at younger ages in whole brain and thalamus, whereas regions with fewer incoming long white matter tracts such as the caudate and putamen would presumably be less influenced by demyelinating injury. Indeed, in the caudate and putamen, the contributions of normal aging and MS-specific atrophy remained stable with age. Future work could test this hypothesis using a similar DTI tractography approach ${ }^{26}$ to delineate incoming and outgoing tracts in the putamen and caudate and to estimate the effect (or lack thereof) of tract-specific white matter lesions on the volume of each structure. If we have indeed identified gray matter regions that are undergoing a high amount of excess MS-specific tissue loss that is relatively independent of white matter lesions, this may open a rich avenue for further exploration that could provide fundamental insights into tissue loss in MS. These regions could be used to study the contribution of other aspects of MS pathology to gray matter atrophy, such as intrinsic gray matter demyelinating lesions, ${ }^{21,22}$ iron deposition, ${ }^{22}$ microglial activation, ${ }^{30}$ mitochondrial dysfunction, ${ }^{31}$ and glutamate excitotoxicity, ${ }^{32}$ which could lead to a more comprehensive understanding of gray matter atrophy in MS.

Our findings may have implications for clinical trial design and interpretation. If we presume that only the MS-specific atrophy is amenable to therapeutic intervention (i.e., that DMTs will not slow normal aging), our data suggest that the most appropriate brain region for a phase II primary end point, as well as the effect size that can reasonably be achieved and the sample size needed to demonstrate efficacy, may differ according to the patient population being studied (early vs late MS, older vs younger patients, etc.). More generally, a better understanding of the time and/or age where MSspecific atrophy is most prominent may help elucidate the "therapeutic opportunity" for current and future DMTs. Our findings could at least partly explain the suggestion in our literature that some DMTs aimed at slowing disease progression may not work as well in older patient populations, ${ }^{33-35}$ perhaps because normal aging is more prominent and there is 
less "therapeutic opportunity" present. Finally, our data support the notion that any "pathologic cutoff" value used to discriminate patients with MS from HCs must be adjusted for age, which has also been suggested by the work of others. ${ }^{5,6}$

Our findings should not suggest that patients with MS at age 60 years are no longer undergoing pathologic disease-related whole-brain or thalamic atrophy. In fact, the total rate of whole-brain and thalamic atrophy in our subjects was consistent throughout the age range at $-0.4 \%$ and $-0.7 \%$ per year, respectively. Rather, our data suggest that normal aging accelerates with age in these regions, which mathematically decreases the excess atrophy because normal aging and MSspecific atrophy were estimated in the same statistical model. Whether whole-brain or regional gray matter atrophy accelerates throughout the MS disease duration has been unclear, with some studies suggesting an acceleration of gray matter atrophy in SPMS compared with RRMS ${ }^{36,37}$ and other, more recent work finding no difference in deep gray matter atrophy rates across MS subtypes. ${ }^{8,38}$ We examined the effects of age rather than clinical subtype; although age and clinical subtype are typically correlated, these should not be conflated. Finally, our findings describe a group-level difference between subjects with MS and HCs. Translating these findings to the individual level is an area of high interest that will require the development of methods to create an MS-specific atrophy biomarker for an individual; exploration of the clinical relevance of such a biomarker is likely to be informative. However, presently, it is premature to apply the current findings at the individual level.

Our approach that focuses on excess, disease-specific atrophy could be applied to other imaging metrics in MS and to other neurodegenerative diseases such as Alzheimer disease, Parkinson disease, and Huntington disease, in which a similar conceptual model should apply. If one could estimate and adjust for the impact of normal aging, disease-specific atrophy measurements could allow for comparison across neurodegenerative diseases, which could uncover biologic, environmental, genetic, and phenotypic similarities (and differences) between diseases that could ultimately further our understanding of neurodegeneration in general. Furthermore, an impact of medical and psychiatric comorbidities on brain atrophy has been demonstrated in both $\mathrm{MS}^{39-41}$ and non$\mathrm{MS}^{42-45}$ subjects. This emerging area deserves further exploration. Because our data sets did not contain information on comorbidities, we were not able to examine their influence on our results. However, all brain regions in a given patient would have been exposed to the same milieu of comorbidities; therefore, it is unlikely that the impact of comorbidities explains our observation of differing behavior between deep gray matter regions. Similarly, the presence or absence of DMT is unlikely to explain our findings, unless one hypothesizes a differential impact of DMT on one deep gray matter structure vs another. Estimating the impact of comorbidities and DMTs on regional brain atrophy in MS will be an area of focus in future studies.
Strengths of this work include the large sample size, longitudinal study design, careful acquisition and postprocessing of MRI data, and the unbiased selection strategy of the top regions of interest, which incorporates normal aging. Our approach of separating normal aging from disease-related atrophy is novel in brain atrophy analyses in the current MS literature and has several implications for MS and other neurodegenerative diseases that could be highly impactful. Limitations include a shorter mean follow-up time in HCs compared with subjects with MS and a relatively small HC sample size. HC MRI data, particularly longitudinal data, are rare in clinical research; efforts to assemble larger $\mathrm{HC}$ data sets are ongoing. However, to achieve the meaningful sample sizes that will be needed to fully understand normal aging will invariably require leveraging and combining multiple existing $\mathrm{HC}$ data sets composed of subjects scanned on different scanners, as we did in this analysis. Although this may initially prompt concern for increasing measurement variability, each $\mathrm{HC}$ subject in this analysis was required to be scanned longitudinally on the same MRI scanner throughout their study period, which reduces variability. ${ }^{15}$ Moreover, we modeled the brain volume changes in this analysis as a percent change from baseline, which obviates the concern regarding heterogeneity across MRI scanners. Finally, by definition, linear mixed-effects models assume a linear decline in the outcome variable (brain volume) over time. Additional work with larger MRI data sets over a much larger age range is underway and will use advanced, nonlinear modeling strategies to further disentangle the contribution of normal aging from the pathologic atrophy observed in MS.

In summary, our findings indicate that the contribution of normal aging may increase with age and the rate of MSspecific atrophy may decrease with age in thalamic and wholebrain atrophy measurements in MS, whereas normal aging and MS-specific atrophy do not change with age in the putamen and caudate. This work emphasizes the need to understand each brain region separately in MS and has many potential implications for MS and perhaps for other neurodegenerative diseases. This could inform future work that furthers our fundamental understanding of brain atrophy in MS, including whether there are different causes of regional tissue loss and how to interpret the effect of disease-modifying therapies with age.

\section{Acknowledgment}

The authors thank the research subjects who generously agreed to serve as study participants, the MS clinicians at the UCSF MS Center who referred patients to the EPIC study, the research coordinators at UCSF for data collection, and the ADNI investigators for the creation of the publicly available ADNI database. All authors had full access to the data and take responsibility for the content of this article.

\section{Study funding}

This study received support from the NIH National Institute of Neurological Disorders and Stroke (R01NS062885 to 
D.P.) for MRI data processing and Biogen and GlaxoSmithKline for MRI data acquisition. This work was also supported by grant UL1TR001855 from the National Center for Advancing Translational Science (NCATS) of the NIH through an Institutional Career Development Award to C.J.A. The content is solely the responsibility of the authors and does not necessarily represent the official views of the National Institutes of Health.

\section{Disclosure}

C.J. Azevedo has received consulting fees from Guerbet, Genentech, Biogen, Novartis, and Sanofi Genzyme. S.Y. Cen, A. Jaberzadeh, and L. Zheng report no disclosures. S.L. Hauser has served on scientific advisory boards for Annexon, Symbiotix, Bionure, and Molecular Stethoscope. He is on the Board of Trustees for Neurona Therapeutics. S.L. Hauser has also received travel reimbursement and writing assistance from F. Hoffman-La Roche Ltd. for CD20-related meetings and presentations. D. Pelletier has received consulting and/or speaking fees from Novartis, Roche, Sanofi Genzyme, and Vertex. Go to Neurology.org/NN for full disclosures.

\section{Publication history}

Received by Neurology: Neuroimmunology \& Neuroinflammation March 16, 2019. Accepted in final form July 1, 2019.

Appendix Authors

\begin{tabular}{|c|c|c|c|}
\hline Name & Location & Role & Contribution \\
\hline $\begin{array}{l}\text { Christina J. } \\
\text { Azevedo, } \\
\text { MD, MPH }\end{array}$ & $\begin{array}{l}\text { University of } \\
\text { Southern } \\
\text { California }\end{array}$ & Author & $\begin{array}{l}\text { Concept and design of the } \\
\text { analysis, data interpretation, } \\
\text { and drafted the manuscript } \\
\text { for intellectual content }\end{array}$ \\
\hline $\begin{array}{l}\text { Steven Y. } \\
\text { Cen, PhD }\end{array}$ & $\begin{array}{l}\text { University of } \\
\text { Southern } \\
\text { California }\end{array}$ & Author & $\begin{array}{l}\text { Performed the statistical } \\
\text { analysis, contributed to the } \\
\text { concept and design of this } \\
\text { analysis, interpretation of } \\
\text { data, and provided critical } \\
\text { revision of the manuscript } \\
\text { for intellectual content }\end{array}$ \\
\hline
\end{tabular}

\begin{tabular}{llcl}
\hline $\begin{array}{l}\text { Amir } \\
\text { Jaberzadeh, } \\
\text { PhD }\end{array}$ & $\begin{array}{l}\text { University of } \\
\text { Southern } \\
\text { California }\end{array}$ & Author & Postprocessing of MRI data \\
\hline $\begin{array}{l}\text { Ling Zheng, } \\
\text { PhD }\end{array}$ & $\begin{array}{l}\text { University of } \\
\text { Southern } \\
\text { California }\end{array}$ & Author & $\begin{array}{l}\text { Provided critical revision of } \\
\text { the manuscript for } \\
\text { intellectual content }\end{array}$ \\
\hline $\begin{array}{l}\text { Stephen L. } \\
\text { Hauser, MD }\end{array}$ & $\begin{array}{l}\text { University of } \\
\text { California, San } \\
\text { Francisco }\end{array}$ & Author & $\begin{array}{l}\text { Provided critical revision of } \\
\text { the manuscript for } \\
\text { intellectual content }\end{array}$ \\
\hline $\begin{array}{l}\text { Daniel } \\
\text { Pelletier, } \\
\text { MD }\end{array}$ & $\begin{array}{l}\text { University of } \\
\text { Southern } \\
\text { California }\end{array}$ & Author & $\begin{array}{l}\text { Concept and design of the } \\
\text { analysis, interpretation of } \\
\text { data, and provided critical } \\
\text { revision of the manuscript } \\
\text { for intellectual content }\end{array}$ \\
\hline & & & \\
\hline
\end{tabular}

\section{References}

1. De Stefano N, Airas L, Grigoriadis N, et al. Clinical relevance of brain volume measures in multiple sclerosis. CNS Drugs 2014;28:147-156.

2. Shiee N, Bazin PL, Zackowski KM, et al. Revisiting brain atrophy and its relationship to disability in multiple sclerosis. PLoS One 2012;7:e37049.
3. Vollmer T, Signorovitch J, Huynh L, et al. The natural history of brain volume loss among patients with multiple sclerosis: a systematic literature review and metaanalysis. J Neurol Sci 2015;357:8-18.

4. Popescu V, Agosta F, Hulst HE, et al. Brain atrophy and lesion load predict long term disability in multiple sclerosis. J Neurol Neurosurg Psychiatry 2013;84: 1082-1091.

5. De Stefano N, Stromillo ML, et al. Establishing pathological cut-offs of brain atrophy rates in multiple sclerosis. J Neurol Neurosurg Psychiatry 2016;87:93-99.

6. Opfer R, Ostwaldt AC, Sormani MP, et al. Estimates of age-dependent cutoffs for pathological brain volume loss using SIENA/FSL—a longitudinal brain volumetry study in healthy adults. Neurobiol Aging 2018;65:1-6.

7. Kappos L, De Stefano N, Freedman MS, et al. Inclusion of brain volume loss in a revised measure of "no evidence of disease activity" (NEDA-4) in relapsingremitting multiple sclerosis. Mult Scler 2016;22:1297-1305.

8. Azevedo CJ, Cen SY, Khadka S, et al. Thalamic atrophy in MS: a magnetic resonance imaging marker of neurodegeneration throughout disease. Ann Neurol 2018;83: 223-234.

9. Walhovd KB, Fjell AM, Reinvang I, et al. Effects of age on volumes of cortex, white matter and subcortical structures. Neurobiol Aging 2005, 26:1261-1268.

10. Fjell AM, Walhovd KB, Fennema-Notestine C, et al. One-year brain atrophy evident in healthy aging. J Neurosci 2009;29:15223-15231.

11. Fjell AM, Walhovd KB. Structural brain changes in aging: courses, causes and cognitive consequences. Rev Neurosci 2010:21:187-221.

12. Hedman AM, van Haren NE, Schnack HG, Kahn RS, Hulshoff Pol HE. Human brain changes across the lifespan: a review of 56 longitudinal magnetic resonance imaging studies. Hum Brain Mapp 2012;33:1987-2002.

13. Polman $\mathrm{CH}$, Reingold SC, Edan G, et al. Diagnostic criteria for multiple sclerosis: 2005 revisions to the "McDonald Criteria. Ann Neurol 2005;58:840-846.

14. Lublin FD, Reingold SC. Defining the clinical course of multiple sclerosis: results of an international survey. National Multiple Sclerosis Society (USA) Advisory Committee on clinical trials of new agents in multiple sclerosis. Neurology 1996; 46:907-911.

15. Josephs KA, Dickson DW, Tosakulwong N, et al. Rates of hippocampal atrophy and post-mortem TDP-43 in Alzheimer's disease: a longitudinal retrospective study. Lancet Neurol 2017;16:917-924.

16. Reuter M, Schmansky NJ, Rosas HD, Fischl B. Within-subject template estimation for unbiased longitudinal image analysis. Neuroimage 2012;61:1402-1418.

17. Sdika M, Pelletier D. Nonrigid registration of multiple sclerosis brain images using lesion inpainting for morphometry or lesion mapping. Hum Brain Mapp 2009;30: 1060-1067.

18. Smith SM, Zhang Y, Jenkinson M, et al. Accurate, robust, and automated longitudinal and cross-sectional brain change analysis. Neuroimage 2002;17:479-489.

19. McCulloch CE, Searle SR, Neuhaus JM. Generalized, Linear, and Mixed Models. Hoboken, NJ: Wiley; 2008.

20. Singer JD. Using SAS PROC MIXED to fit multilevel models, hierarchical models, and individual growth models. J Educ Behav Stat 1998;23:323-355.

21. Vercellino M, Masera S, Lorenzatti $M$, et al. Demyelination, inflammation, and neurodegeneration in multiple sclerosis deep gray matter. J Neuropathol Exp Neurol 2009;68:489-502.

22. Haider L, Simeonidou C, Steinberger G, et al. Multiple sclerosis deep grey matter: the relation between demyelination, neurodegeneration, inflammation and iron. J Neurol Neurosurg Psychiatry 2014;85:1386-1395.

23. Peterson JW, Bö L, Mörk S, Chang A, Trapp BD. Transected neurites, apoptotic neurons, and reduced inflammation in cortical multiple sclerosis lesions. Ann Neurol 2001;50:389-400.

24. Trapp BD, Peterson J, Ransohoff RM, Rudick R, Mörk S. Bö L. Axonal transection in the lesions of multiple sclerosis. N Engl J Med 1998;338:278-285.

25. Bermel RA, Bakshi R. The measurement and clinical relevance of brain atrophy in multiple sclerosis. Lancet Neurol 2005;5:158-170.

26. Henry RG, Shieh M, Amirbekian B, Chung S, Okuda DT, Pelletier D. Connecting white matter injury and thalamic atrophy in clinically isolated syndromes. J Neurol Sci 2009;282:61-66.

27. Aubert-Broche B, Fonov V, Ghassemi R, et al. Regional brain atrophy in children with multiple sclerosis. Neuroimage 2011;58:409-415.

28. Kolasinski J, Stagg CJ, Chance SA, et al. A combined post-mortem magnetic resonance imaging and quantitative histological study of multiple sclerosis pathology. Brain 2012:135;2938-2951.

29. Azevedo CJ, Overton E, Khadka S, et al. Early CNS neurodegeneration in radiologically isolated syndrome. Neurol Neuroimmunol Neuroinflamm 2015;2:3.

30. Lassmann H. Multiple sclerosis: lessons from molecular neuropathology. Exp Neurol 2014;262:2-7.

31. Witte ME, Mahad DJ, Lassmann H, van Horssen J. Mitochondrial dysfunction contributes to neurodegeneration in multiple sclerosis. Trends Mol Med 2014;20: 179-187.

32. Matute C, Domercq M, Sánchez-Gómez MV. Glutamate-mediated glial injury: mechanisms and clinical importance. Glia 2006;53:212-224.

33. Hawker K, O'Connor P, Freedman MS, et al. Rituximab in patients with primary progressive multiple sclerosis: results of a randomized double-blind placebocontrolled multicenter trial. Ann Neurol 2009;66:460-471.

34. La Mantia L, Vacchi L, Rovaris M, et al. Interferon $\beta$ for secondary progressive multiple sclerosis: a systematic review. J Neurol Neurosurg Psychiatry 2013;84:420-426.

35. Segal BM, Stuve O. Primary progressive multiple sclerosis-why we are failing. Lancet 2016;387:1032-1034. 
36. Fisher E, Lee JC, Nakamura K, Rudick RA. Gray matter atrophy in multiple sclerosis: a longitudinal study. Ann Neurol 2008;64:255-265.

37. Fisniku LK, Chard DT, Jackson JS, et al. Gray matter atrophy is related to long-term disability in multiple sclerosis. Ann Neurol 2008;64:247-254.

38. Eshaghi A, Prados F, Brownlee WJ, et al. Deep gray matter volume loss drives disability worsening in multiple sclerosis. Ann Neurol 2018;83:210-222.

39. Kappus N, Weinstock-Guttman B, Hagemeier J, et al. Cardiovascular risk factors are associated with increased lesion burden and brain atrophy in multiple sclerosis. J Neurol Neurosurg Psychiatry 2016;87:181-187.

40. Weinstock-Guttman B, Zivadinov R, Mahfooz N, et al. Serum lipid profiles are associated with disability and MRI outcomes in multiple sclerosis. J Neuroinflammation $2011 ; 8: 127$.
41. Mowry EM, Azevedo CJ, McCulloch CE, et al. Body mass index, but not vitamin D status, is associated with brain volume change in MS. Neurology 2018;91: e2256-e2264.

42. Torelli F, Moscufo N, Garreffa G, et al. Cognitive profile and brain morphologica changes in obstructive sleep apnea. Neuroimage 2011;54:787-793.

43. Raji CA, Ho AJ, Parikshak NN, et al. Brain structure and obesity. Hum Brain Mapp 2010;31:353-364.

44. Pan P, Shi H, Zhong J, et al. Chronic smoking and brain gray matter changes: evidence from meta-analysis of voxel-based morphometry studies. Neurol Sci 2013;34:813-817.

45. Bruehl H, Wolf OT, Sweat V, Tirsi A, Richardson S, Convit A. Modifiers of cognitive function and brain structure in middle-aged and elderly individuals with type 2 diabetes mellitus. Brain Res 2009;1280:186-194. 


\section{Neurology \\ Neuroimmunology \& Neuroinflammation}

Contribution of normal aging to brain atrophy in MS Christina J. Azevedo, Steven Y. Cen, Amir Jaberzadeh, et al.

Neurol Neuroimmunol Neuroinflamm 2019;6;

DOI 10.1212/NXI.0000000000000616

This information is current as of September 25, 2019

Neurol Neuroimmunol Neuroinflamm is an official journal of the American Academy of Neurology.

Published since April 2014, it is an open-access, online-only, continuous publication journal. Copyright

Copyright $\odot 2019$ The Author(s). Published by Wolters Kluwer Health, Inc. on behalf of the American Academy of Neurology.. All rights reserved. Online ISSN: 2332-7812.

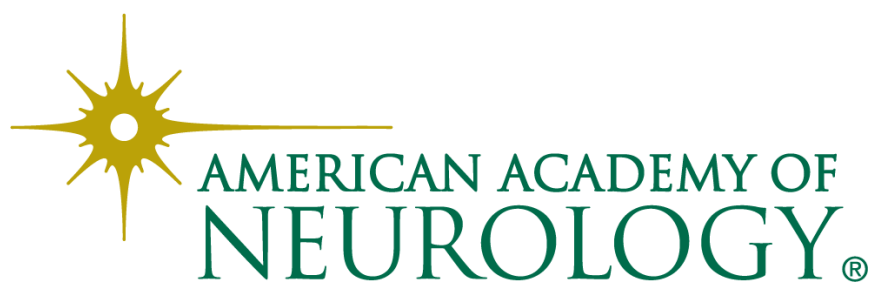




\section{Updated Information \& Services}

References

Citations

Subspecialty Collections

Permissions \& Licensing

Reprints including high resolution figures, can be found at: http://nn.neurology.org/content/6/6/e616.full.html

This article cites 44 articles, 6 of which you can access for free at: http://nn.neurology.org/content/6/6/e616.full.html\#\#ref-list-1

This article has been cited by 8 HighWire-hosted articles: http://nn.neurology.org/content/6/6/e616.full.html\#\#otherarticles

This article, along with others on similar topics, appears in the following collection(s):

All Demyelinating disease (CNS)

http://nn.neurology.org//cgi/collection/all_demyelinating_disease_cns MRI

http://nn.neurology.org//cgi/collection/mri

Multiple sclerosis

http://nn.neurology.org//cgi/collection/multiple_sclerosis

Volumetric MRI

http://nn.neurology.org//cgi/collection/volumetric_mri

Information about reproducing this article in parts (figures,tables) or in its entirety can be found online at:

http://nn.neurology.org/misc/about.xhtml\#permissions

Information about ordering reprints can be found online:

http://nn.neurology.org/misc/addir.xhtml\#reprintsus

Neurol Neuroimmunol Neuroinflamm is an official journal of the American Academy of Neurology.

Published since April 2014, it is an open-access, online-only, continuous publication journal. Copyright

Copyright $\odot 2019$ The Author(s). Published by Wolters Kluwer Health, Inc. on behalf of the American

Academy of Neurology.. All rights reserved. Online ISSN: 2332-7812.

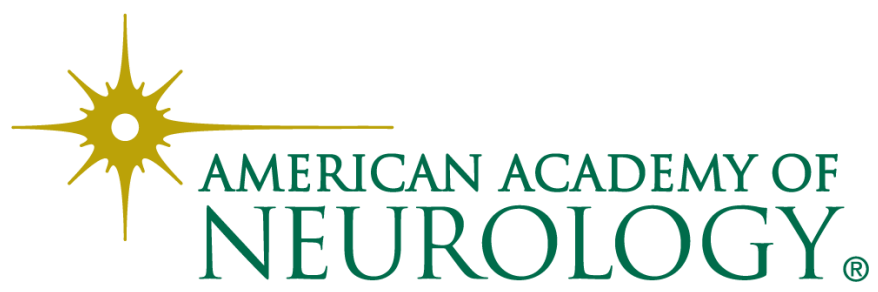

